\title{
ANÁLISE DE APLICATIVOS MÓVEIS PARA CUIDADORES DE IDOSOS EM PLATAFORMAS DIGITAIS
}

\author{
Jocênio Marquios Epaminondas ${ }^{1}$ \\ Ana Catarine Melo de Oliveira Carneiro² \\ Rodrigo Alexandre Félix ${ }^{3}$ \\ Karla Helena Coelho Vilaça e Silva ${ }^{4}$
}

resumo

O objetivo deste estudo foi analisar aplicativos móveis disponíveis nas plataformas Play Store e App Store para cuidadores de idosos no Brasil. Foram analisados aplicativos na língua portuguesa que atuam como instrumentos de auxílio no cuidado ao idoso e/ou que promova a relação comercial entre cuidador de idoso profissional e família. Como resultado da busca, foram encontrados 247 aplicativos

1 Licenciado em Informática. Doutorando em Gerontologia na UCB. Professor do Instituto Federal de Brasília (IFB). E-mail: jocenio.epaminondas@ifb.edu.br.

2 Graduada e licenciada em Enfermagem. Mestranda em Gerontologia na UCB. Enfermeira da Secretaria de Estado de Saúde do Distrito Federal. E-mail: anacatarine@gmail.com.

3 Graduado em Odontologia. Mestrando em Gerontologia na UCB. Professor de Odontologia Hospitalar e Intensiva no Hospital Daher e de implantodontia no Instituto Ária. E-mail: rodrigoafelix@ gmail.com.

4 Graduada em Fisioterapia. Doutora em Investigação Biomédica pela FMRP-USP. Professora do Programa de Pós-Graduação Stricto Sensu em Gerontologia da Universidade Católica de Brasília (UCB). E-mail: karlav@ucb.br. 
que se encaixassem nos perímetros da pesquisa. Após critérios de exclusão, restaram 28 aplicativos que foram organizados em categorias, sendo que dezoito aplicativos apresentaram função de aproximar profissionais/serviços e familiares/idosos, sete para auxílio ao idoso, cuidador profissional ou familiar, três para monitoramento do idoso, quatro para registro de informações do idoso e cinco pertencem a mais de uma categoria. Conclui-se que há aplicativos disponíveis nas lojas virtuais com temas voltados para o cuidado do idoso e que promovem relacionamento entre os familiares e o idoso assistido. Todavia, evidenciou-se a necessidade de inclusão de novas funcionalidades e ajustes para melhoria da usabilidade dos aplicativos.

palavras-chave

Avaliação da Tecnologia Biomédica. Aplicativos Móveis. Cuidadores. Idoso.

\section{Introdução}

A introdução do dispositivo móvel nos anos noventa permitiu que profissionais acessassem facilmente prontuários de pacientes, resultados de exames, imagens e informações sobre medicamentos e tratamentos (SILVA et al., 2015). Atualmente, a evolução tecnológica e a facilidade de acesso à tecnologia nos mostram um número maior de dispositivos móveis do que os habitantes no planeta, sendo o Smartphone o dispositivo móvel mais utilizado no mundo (MEIRELLES, 2019).

Esses dispositivos móveis proporcionam diversas vantagens, a destacar: armazenamento e compartilhamento de informações, maior relacionamento entre os profissionais envolvidos e seus usuários, acesso facilitado aos dados, navegação na Rede Mundial de Computadores (Internet), troca de mensagens eletrônicas (como o e-mail), acesso a redes sem fios, dentre outros. Outra vantagem desses dispositivos é que eles servem de repositório para a instalação de aplicativos móveis (WAISBOURD et al., 2016). Seguindo essa vertente, Kim (2017) aponta que a Internet fornece um amplo acesso às informações sobre saúde e permite que pessoas interajam entre si e compartilhem informações. Nesse contexto, observa-se um aumento exponencial no número de idosos cuja projeção em 2050 é de 66,5 milhões de pessoas, ou seja, 29,3\% da população brasileira (IBGE, 2010). Esse fenômeno impacta diretamente na crescente 
demanda por serviços de saúde e cuidados aos idosos, que têm como objetivo mantê-los autônomos e independentes para a realização das atividades do cotidiano (REIS; NOVELLI; GUERRA, 2018).

Estudos anteriores têm demonstrado que os aplicativos móveis possuem benefícios como o fornecimento de recursos e informações para a população, que permitem rápida interação com profissionais de saúde, proporcionam a compreensão de fatores determinantes que promovem a saúde, auxiliam no monitoramento e no gerenciamento de problemas com idosos e familiares e podem reduzir a sobrecarga e o estresse dos cuidadores (BARRA et al., 2017; GROSSMAN; ZAK; ZELINSKI, 2018).

Estudo recente realizado nos Estados Unidos evidenciou que o uso da tecnologia é desejável para a população de idosos e cuidadores (QUINN et al., 2019). Sendo assim, garantir o bem-estar pessoal por meio de aplicativos para smartphones, em uma população cada vez mais envelhecida, exige o monitoramento de vários comportamentos nas dimensões física, mental e social (BRAVO et al., 2018).

Para garantir maior eficácia, aumento da receptividade e maior adesão, é necessário reconhecer as opiniões dos usuários. Os cuidadores expressam a importância de se ter um cuidado em relação à segurança e usabilidade ao projetar aplicativos móveis (PHONGTANKUEL et al, 2018). Também é importante dispor de mecanismos que ofereçam segurança ao idoso no momento da contratação do serviço, por exemplo: verificação de antecedentes criminais, qualificação técnica do profissional, registro funcional, carta de referência, etc. Dessa forma, este trabalho justifica-se pelo aumento da população idosa e da necessidade de estudos que avaliem e aprimorem o uso da tecnologia utilizada para benefício dessa população. Nesse contexto, este trabalho tem como objetivo analisar os aplicativos móveis disponíveis nas plataformas Play Store e App Store para cuidadores de idosos no Brasil e, assim, apresentar informações, relacionar as funcionalidades, apresentar melhorias e identificar o que é ofertado em comum pelos aplicativos.

\section{Métodos}

Realizou-se busca de aplicativos voltados para cuidadores de idosos em smartphones com sistemas operacionais Android, na Google Play Store, e iOS, na Apple Store, no mês de setembro de 2019. No campo de busca dessas lojas virtuais, foram utilizados os termos, sem aspas, "Cuidador de idoso", na Play Store, e "Cuidador" e "Idoso", na Apple Store. Essa divergência ocorreu, pois, 
nessa última, não foram encontrados resultados com o termo "Cuidador de idoso", na Apple Store. Na busca realizada na Google Play Store - feita por um celular com sistema operacional Android -, foram encontrados 247 aplicativos por meio da busca pelo termo "Cuidador de idoso". Na Apple Store, foram encontrados dezessete aplicativos com a palavra "Cuidador" e 21 aplicativos com a palavra "Idoso".

Os aplicativos encontrados nas buscas foram armazenados em planilhas eletrônicas para serem igualmente avaliados por três dos pesquisadores simultaneamente. Os pesquisadores fizeram avaliações distintas dos aplicativos. Por meio da leitura de suas descrições, os artigos foram excluídos pelos critérios: estar em outros idiomas, ser pago, tratar de uma doença específica, não ser específico para idosos, ter única função de gerenciamento de medicamentos, ter acesso antecipado, ser restrito a apenas um estado brasileiro e/ou ser voltado ao diagnóstico e à avaliação em saúde por profissionais de saúde. Os demais foram baixados pelos pesquisadores para confirmar os critérios de inclusão - aplicativos com orientações e/ou instrumentos de auxílio para o cuidado do idoso e/ou que promovam a relação comercial entre o cuidador profissional do idoso e a família - e diferenciá-los pela sua principal função. As divergências na avaliação foram debatidas pelos pesquisadores. A partir desses critérios, restaram 28 aplicativos que foram organizados em categorias: prestador de serviço, familiares e idosos; para auxílio ao idoso, cuidador profissional ou familiar; monitoramento do idoso; e para registro de informações do idoso.

\section{Resultados e Discussão}

Após a análise, foram selecionados ao todo 28 aplicativos que foram organizados por categoria conforme a principal funcionalidade. Sete são ferramentas de auxílio para o idoso ou cuidador profissional ou familiar. Como ferramentas de monitoramento, há quatro aplicativos. Dezoito tiveram como objetivo conectar profissionais/serviços e familiares/idosos. Por fim, como espaço para registro de informações sobre o idoso, foram localizados quatro aplicativos. Ao total, cinco pertencem a mais de uma categoria. Cada categoria é apresentada nos Quadros a seguir, com as informações dos aplicativos selecionados. 
Quadro 1 - Categoria: auxílio para o idoso, cuidador familiar ou profissional.

\begin{tabular}{|c|c|c|c|c|}
\hline Aplicativo & $\begin{array}{l}\text { Sistema } \\
\text { Operacional }\end{array}$ & $\begin{array}{l}\text { Termo } \\
\text { para busca }\end{array}$ & $\begin{array}{l}\text { Avaliação na } \\
\text { Plataforma }\end{array}$ & Funcionalidades \\
\hline $\begin{array}{l}\text { Cuidador } \\
\text { Familiar }\end{array}$ & Android & $\begin{array}{l}\text { Cuidador de } \\
\text { idoso }\end{array}$ & 5,0 & $\begin{array}{l}\text { Promove rede de apoio } \\
\text { entre cuidadores familiares } \\
\text { e grupos de acordo com o } \\
\text { idoso. } \\
\text { Disponibiliza informações } \\
\text { sobre a saúde dos idosos } \\
\text { por diferentes meios. } \\
\text { Conecta profissionais a } \\
\text { familiares. }\end{array}$ \\
\hline $\begin{array}{l}\text { Cuidador de } \\
\text { Idosos }\end{array}$ & Android & $\begin{array}{l}\text { Cuidador de } \\
\text { idoso }\end{array}$ & 4,5 & $\begin{array}{l}\text { Aponta escolas de } \\
\text { cuidadores. } \\
\text { Auxilia na organização de } \\
\text { plantões e cobrança de } \\
\text { valor. } \\
\text { Direciona ligação para } \\
\text { emergências. } \\
\text { Disponibiliza profissionais } \\
\text { para contratação. }\end{array}$ \\
\hline Elderly Care & Android & $\begin{array}{l}\text { Cuidador de } \\
\text { idoso }\end{array}$ & 4,4 & $\begin{array}{l}\text { Oferece informações e } \\
\text { notícias. }\end{array}$ \\
\hline $\begin{array}{l}\text { Cuidando do } \\
\text { Idoso - saúde, } \\
\text { proteção e } \\
\text { felicidade }\end{array}$ & Android & $\begin{array}{l}\text { Cuidador de } \\
\text { Idoso }\end{array}$ & 4,0 & $\begin{array}{l}\text { Apresenta sites seguros } \\
\text { para pesquisas. Emite infor- } \\
\text { mações do celular do idoso } \\
\text { para cuidadores. }\end{array}$ \\
\hline $\begin{array}{l}\text { Não deixe a } \\
\text { Vovó cair }\end{array}$ & Android & $\begin{array}{l}\text { Cuidador de } \\
\text { idoso }\end{array}$ & 3,8 & $\begin{array}{l}\text { Orienta sobre cuidados } \\
\text { ambientais para reduzir risco } \\
\text { de queda }\end{array}$ \\
\hline Idoso Ativo & iOS/ Android & $\begin{array}{l}\text { Idoso (iOS) } \\
\text { Cuidador } \\
\text { de ldoso } \\
\text { (Android) }\end{array}$ & 3,0 & $\begin{array}{l}\text { Fornece programa de } \\
\text { exercícios funcionais para } \\
\text { o idoso. }\end{array}$ \\
\hline Easierphone & iOs & Idoso & - & $\begin{array}{l}\text { Facilita o uso do celular pelo } \\
\text { idoso. } \\
\text { Emite informações do } \\
\text { celular do idoso para } \\
\text { cuidadores. }\end{array}$ \\
\hline
\end{tabular}

Fonte: Elaborado pelos autores. 
Quadro 2 - Categoria: monitoramento.

\begin{tabular}{|l|l|l|l|l|}
\hline Aplicativo & $\begin{array}{l}\text { Sistema } \\
\text { Operacional }\end{array}$ & $\begin{array}{l}\text { Termo } \\
\text { para busca }\end{array}$ & $\begin{array}{l}\text { Avaliação na } \\
\text { Plataforma }\end{array}$ & Funcionalidade \\
\hline LifeStars & $\begin{array}{l}\text { iOS e } \\
\text { Android }\end{array}$ & $\begin{array}{l}\text { Cuidador } \\
\text { (iOS) } \\
\text { Cuidador } \\
\text { de idoso } \\
\text { (Android) }\end{array}$ & 5,0 & $\begin{array}{l}\text { Disponibilidade de profissio- } \\
\text { nais para contratação. } \\
\text { Monitoramento de ativi- } \\
\text { dades na residência em } \\
\text { tempo real. }\end{array}$ \\
\hline $\begin{array}{l}\text { Cuidando } \\
\text { do Idoso } \\
- \text { saúde, } \\
\text { proteção } \\
\text { felicidade }\end{array}$ & Android & $\begin{array}{l}\text { Cuidador de } \\
\text { idoso }\end{array}$ & 4,0 & $\begin{array}{l}\text { Apresenta sites seguros } \\
\text { para pesquisas. Emite infor- } \\
\text { mações do celular do idoso } \\
\text { para cuidadores. }\end{array}$ \\
\hline $\begin{array}{l}\text { MonitoriQ } \\
- \text { Monitora- } \\
\text { mento de } \\
\text { idosos }\end{array}$ & Android & $\begin{array}{l}\text { Cuidador de } \\
\text { idoso }\end{array}$ & 3,7 & $\begin{array}{l}\text { Emite informações do } \\
\text { celular do idoso para } \\
\text { cuidadores. }\end{array}$ \\
\hline $\begin{array}{l}\text { Easierphone } \\
\text { ildososo }\end{array}$ & Idoso & - & $\begin{array}{l}\text { Facilita o uso do celular } \\
\text { pelo idoso. } \\
\text { Emite informações do } \\
\text { celular do idoso para } \\
\text { cuidadores. }\end{array}$ \\
\hline
\end{tabular}

Fonte: Elaborado pelos autores.

Quadro 3 - Categoria: conectar serviços/profissionais e idosos.

\begin{tabular}{|l|l|l|l|l|}
\hline Aplicativo & $\begin{array}{l}\text { Sistema } \\
\text { Operacional }\end{array}$ & $\begin{array}{l}\text { Termo } \\
\text { para busca }\end{array}$ & $\begin{array}{l}\text { Avaliação na } \\
\text { Plataforma }\end{array}$ & Funcionalidade \\
\hline $\begin{array}{l}\text { Cuidador } \\
\text { Familiar }\end{array}$ & Android & $\begin{array}{l}\text { Cuidador de } \\
\text { idoso }\end{array}$ & 5,0 & $\begin{array}{l}\text { Promove rede de apoio } \\
\text { entre cuidadores familiares } \\
\text { e grupos. } \\
\text { Disponibiliza informações } \\
\text { sobre a saúde dos idosos } \\
\text { por diferentes meios. } \\
\text { Conecta profissionais a } \\
\text { familiares. }\end{array}$ \\
\hline Hugs Care & iOS & $\begin{array}{l}\text { Cuidador de } \\
\text { idoso }\end{array}$ & 5,0 & $\begin{array}{l}\text { Disponibiliza contatos } \\
\text { de profissionais para } \\
\text { contratação }\end{array}$ \\
\hline LifeStars & $\begin{array}{l}\text { iOS e } \\
\text { Android }\end{array}$ & $\begin{array}{l}\text { Cuidador } \\
\text { (iOS) } \\
\text { Cuidador } \\
\text { de idoso } \\
\text { (Android) }\end{array}$ & 5,0 & $\begin{array}{l}\text { Disponibiliza profissionais } \\
\text { para contratação. } \\
\text { Monitora as atividades na } \\
\text { residência em tempo real. }\end{array}$ \\
\hline
\end{tabular}




\begin{tabular}{|c|c|c|c|c|}
\hline Aplicativo & $\begin{array}{l}\text { Sistema } \\
\text { Operacional }\end{array}$ & $\begin{array}{l}\text { Termo } \\
\text { para busca }\end{array}$ & $\begin{array}{l}\text { Avaliação na } \\
\text { Plataforma }\end{array}$ & Funcionalidade \\
\hline $\begin{array}{l}\text { Icuidador } \\
\text { - familiar }\end{array}$ & $\begin{array}{l}\text { iOS e } \\
\text { Android }\end{array}$ & $\begin{array}{l}\text { Cuidador } \\
\text { (iOS) } \\
\text { Cuidador } \\
\text { de idoso } \\
\text { (Android) }\end{array}$ & 5,0 & $\begin{array}{l}\text { Disponibiliza profissionais } \\
\text { para contratação. }\end{array}$ \\
\hline $\begin{array}{l}\text { Cuidador de } \\
\text { idosos }\end{array}$ & Android & $\begin{array}{l}\text { Cuidador de } \\
\text { idoso }\end{array}$ & 4,5 & $\begin{array}{l}\text { Aponta escolas de } \\
\text { cuidadores. } \\
\text { Auxilia a organização de } \\
\text { plantões e cobrança de } \\
\text { valor. } \\
\text { Direciona ligação para } \\
\text { emergências. } \\
\text { Disponibiliza profissionais } \\
\text { para contratação. }\end{array}$ \\
\hline $\begin{array}{l}\text { Perfil do } \\
\text { Cuidador }\end{array}$ & Android & $\begin{array}{l}\text { Cuidador de } \\
\text { idoso }\end{array}$ & 4,3 & $\begin{array}{l}\text { Oferece cadastro de profis- } \\
\text { sionais da área da saúde } \\
\text { e cuidadores em geral, } \\
\text { desenvolvendo trabalho } \\
\text { home care. }\end{array}$ \\
\hline $\begin{array}{l}\text { KDCARE } \\
\text { Profissional }\end{array}$ & $\begin{array}{l}\text { iOS e } \\
\text { Android }\end{array}$ & $\begin{array}{l}\text { Cuidador } \\
\text { (iOS) } \\
\text { Cuidador } \\
\text { de idoso } \\
\text { (Android) }\end{array}$ & $\begin{array}{l}\text { 4,0 (iOS) } \\
\text { 3,4 (Android) }\end{array}$ & $\begin{array}{l}\text { Disponibiliza profissionais } \\
\text { para contratação. }\end{array}$ \\
\hline KDCare & $\begin{array}{l}\text { iOS e } \\
\text { Android }\end{array}$ & $\begin{array}{l}\text { Cuidador } \\
\text { (iOS) } \\
\text { Cuidador } \\
\text { de idoso } \\
\text { (Android) }\end{array}$ & $\begin{array}{l}\text { 4,0 (iOS) } \\
3,6 \text { (Android) }\end{array}$ & $\begin{array}{l}\text { Disponibilidade de profis- } \\
\text { sionais para contratação. }\end{array}$ \\
\hline $\begin{array}{l}\text { Ache um } \\
\text { Cuidador }\end{array}$ & Android & $\begin{array}{l}\text { Cuidador de } \\
\text { idoso }\end{array}$ & 3,7 & $\begin{array}{l}\text { Disponibiliza profissionais } \\
\text { para contratação. }\end{array}$ \\
\hline Cuidar Idoso & Android & $\begin{array}{l}\text { Cuidador de } \\
\text { idoso }\end{array}$ & 3,2 & $\begin{array}{l}\text { Disponibiliza profissionais } \\
\text { para contratação. }\end{array}$ \\
\hline $\begin{array}{l}\text { Easy Care } \\
\text { Saúde }\end{array}$ & $\begin{array}{l}\text { iOS e } \\
\text { Android }\end{array}$ & $\begin{array}{l}\text { Cuidador } \\
\text { (iOS) } \\
\text { Cuidador } \\
\text { de idoso } \\
\text { (Android) }\end{array}$ & $\begin{array}{l}\text { 3,0 (iOS) } \\
\text { 2,7 (Android) }\end{array}$ & $\begin{array}{l}\text { Disponibiliza profissionais } \\
\text { para contratação. }\end{array}$ \\
\hline Helpmy & Android & $\begin{array}{l}\text { Cuidador de } \\
\text { idoso }\end{array}$ & 2,6 & $\begin{array}{l}\text { Disponibiliza profissionais } \\
\text { para contratação. }\end{array}$ \\
\hline
\end{tabular}




\begin{tabular}{|l|l|l|l|l|}
\hline Aplicativo & $\begin{array}{l}\text { Sistema } \\
\text { Operacional }\end{array}$ & $\begin{array}{l}\text { Termo } \\
\text { para busca }\end{array}$ & $\begin{array}{l}\text { Avaliação na } \\
\text { Plataforma }\end{array}$ & Funcionalidade \\
\hline $\begin{array}{l}\text { iCuidador } \\
- \text { prestador }\end{array}$ & $\begin{array}{l}\text { iOS e } \\
\text { Android }\end{array}$ & $\begin{array}{l}\text { Cuidador } \\
\text { (iOS) } \\
\text { Cuidador } \\
\text { de idoso } \\
\text { (Android) }\end{array}$ & $\begin{array}{l}- \text { OS } \\
2,5 \text { (Android) }\end{array}$ & $\begin{array}{l}\text { Disponibiliza profissionais } \\
\text { para contratação. }\end{array}$ \\
\hline $\begin{array}{l}\text { Helpmy } \\
\text { Profissionais }\end{array}$ & Android & $\begin{array}{l}\text { Cuidador de } \\
\text { idoso }\end{array}$ & 1,3 & $\begin{array}{l}\text { Disponibilidade de profis- } \\
\text { sionais para contratação. }\end{array}$ \\
\hline $\begin{array}{l}\text { Cuidadores } \\
\text { de ldoso no } \\
\text { Brasil Inteiro }\end{array}$ & Android & $\begin{array}{l}\text { Cuidador de } \\
\text { idoso }\end{array}$ & - & $\begin{array}{l}\text { Disponibiliza profissionais } \\
\text { para contratação. }\end{array}$ \\
\hline $\begin{array}{l}\text { Diário } \\
\text { Cuidador }\end{array}$ & Android & $\begin{array}{l}\text { Cuidador de } \\
\text { idoso }\end{array}$ & - & $\begin{array}{l}\text { Disponibiliza profissionais } \\
\text { para contratação. }\end{array}$ \\
\hline $\begin{array}{l}\text { Life Senior } \\
\text { Cuidador de }\end{array}$ & Android & - & $\begin{array}{l}\text { Disponibiliza profissionais } \\
\text { para contratação em hospi- } \\
\text { tais ou residência. }\end{array}$ \\
\hline $\begin{array}{l}\text { Mais Amor } \\
\text { Cuidadores }\end{array}$ & Android & $\begin{array}{l}\text { Cuidador de } \\
\text { idoso }\end{array}$ & - & Disponibiliza profissionais \\
\hline
\end{tabular}

Fonte: Elaborado pelos autores.

Quadro 4 - Categoria: registro de informações.

\begin{tabular}{|l|l|l|l|l|}
\hline Aplicativo & $\begin{array}{l}\text { Sistema } \\
\text { Operacional }\end{array}$ & $\begin{array}{l}\text { Termo para } \\
\text { busca }\end{array}$ & $\begin{array}{l}\text { Avaliação na } \\
\text { Plataforma }\end{array}$ & Funcionalidade \\
\hline Plug and Care & $\begin{array}{l}\text { OS e } \\
\text { Android }\end{array}$ & $\begin{array}{l}\text { Cuidador } \\
\text { (iOS) } \\
\text { Cuidador } \\
\text { de idoso } \\
\text { (Android) }\end{array}$ & $\begin{array}{l}5,0 \text { (iOS) } \\
3,6 \text { (Android) }\end{array}$ & $\begin{array}{l}\text { Gerencia medicamentos, } \\
\text { sinais vitais, eventos e } \\
\text { círculo de cuidado. }\end{array}$ \\
\hline $\begin{array}{l}\text { Saúde em dia } \\
\text { - Gerenciador } \\
\text { de saúde } \\
\text { familiar }\end{array}$ & Android & $\begin{array}{l}\text { Cuidador de } \\
\text { idoso }\end{array}$ & 4,9 & $\begin{array}{l}\text { Gerencia medicamentos, } \\
\text { consultas, alergias, dentre } \\
\text { outros. }\end{array}$ \\
\hline $\begin{array}{l}\text { Gero 360 } \\
\text { iOS }\end{array}$ & $\begin{array}{l}\text { Idoso (iOS) } \\
\text { Cuidador de } \\
\text { idoso (iOS) }\end{array}$ & 4,8 & $\begin{array}{l}\text { Gerencia medicamentos, } \\
\text { sinais vitais, eventos e } \\
\text { círculo de cuidado. }\end{array}$ \\
\hline Cori Saúde & iOS e & $\begin{array}{l}\text { Cuidador } \\
\text { (iOS) } \\
\text { Cuidador } \\
\text { de idoso } \\
\text { (Android) }\end{array}$ & $\begin{array}{l}\text { - iOS } \\
4,4 \text { (Android) }\end{array}$ & $\begin{array}{l}\text { Gerencia medicamentos, } \\
\text { compromissos, sinais } \\
\text { vitais e desempenho em } \\
\text { atividades de vida diária. } \\
\text { Disponibiliza escalas de } \\
\text { avaliação. }\end{array}$ \\
\hline
\end{tabular}

Fonte: Elaborado pelos autores. 
A maior parte (57,14\%) dos aplicativos são desenvolvidos apenas para o sistema operacional Android, seguido de 32,14\% para ambos (Android e iOS) e apenas $10,71 \%$ exclusivamente na plataforma $i O S$ (Figura 1).

Figura 1 - Percentual de Aplicativos por Sistema Operacional.

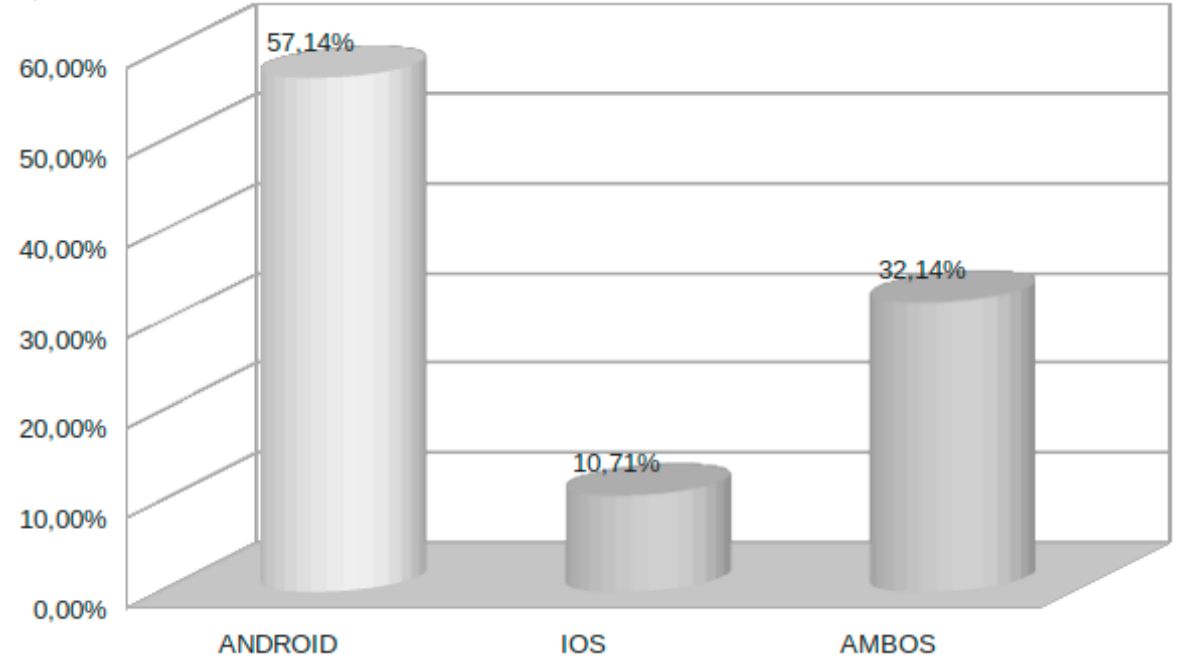

Fonte: Elaborada pelos autores.

Observou-se, portanto, que o maior número dos aplicativos é criado para a plataforma Android, o sistema operacional da maioria dos celulares no mundo (GARTNER, 2017). A busca de aplicativos pela plataforma Android, porém, demonstrou ser menos específica. Resultou em vários aplicativos sem relação com o tema, o que representa obstáculo ao encontro de aplicativos adequados à necessidade do usuário. De acordo com a Figura 2, a maioria dos aplicativos (67,22\%) foi avaliada com pontuação maior ou igual a 4 . Alguns aplicativos, contudo, possuem pontuação intermediária (22,80\%) e outros não foram avaliados ou não tiveram uma boa avaliação $(9,98 \%)$. 
Figura 2 - Avaliação dos aplicativos.

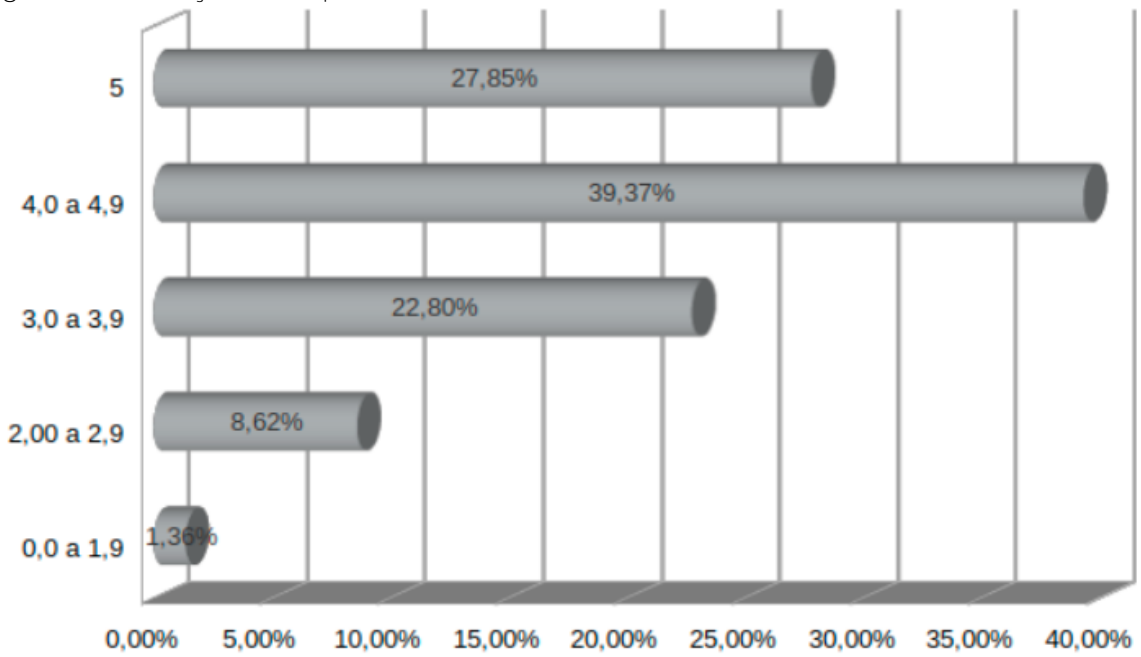

Fonte: Elaborada pelos autores.

O aplicativo com maior número de avaliações e pontuação na plataforma é o "Cuidador Familiar", que permite a interação entre cuidadores e profissionais e fornece informações sobre saúde por diferentes meios digitais. Exceto pela função de monitoramento e registro, esse aplicativo corresponde à expectativa dos usuários descritos em estudos. Quinn et al. (2019) contam que cuidadores de idosos relataram querer usar aplicativos para registrar informações sobre o idoso, compartilharem informações sobre as condições clínicas e estarem mais engajados sobre a saúde dos idosos, além de terem acesso às informações em um só lugar.

Williamson, Gorman e Jimison (2014) relatam que, para os cuidadores, as informações mais necessárias para o registro em aplicativos são sobre administração e adesão de medicamentos, calendário e saúde cognitiva. Além dessas funcionalidades, outras foram apontadas como chamadas de vídeo, dados de atividades relacionadas ao sono e exercício físico, comunicação assíncrona, compartilhamento de fotos, espaço para registro das informações em diário ou prontuário, acesso a recursos de saúde on-line, monitoramento em tempo real, possibilidade de ter um resumo geral da saúde e sugestões para ajudá-los a melhorar como cuidadores.

Já o estudo de Warpenius et al. (2015), identificou que os recursos mais procurados na perspectiva dos familiares foram relatórios de intercorrência, alarmes, agenda de visitas ao médico, avaliação das condições gerais do idoso 
simplicidade e facilidade de uso. Esses recursos são menos encontrados nos aplicativos, conforme apresentado nos Quadros anteriores. Contudo, são encontrados relatos semelhantes nos comentários dos usuários desses recursos.

Ademais, salienta-se que o usuário brasileiro relata a importância dos aplicativos na vida profissional e no cuidado ao idoso e na aproximação com seus familiares. Por ser importante na área da gerontologia, é fundamental que os aplicativos forneçam a possibilidade de conectar o idoso a uma rede de apoio, ao cuidador familiar e aos profissionais.

Apesar da preferência dos usuários por outras funções, a maior parte dos aplicativos (64\%) oferece a categoria "conectar serviços/profissionais e idosos". Destaca-se ainda que esses aplicativos não oferecem recursos para que os contratantes possam ter acesso ao histórico profissional, como referências, qualificação técnica, registro funcional e/ou antecedentes criminais dos profissionais, aumentando a insegurança de idosos e familiares na escolha do cuidador profissional. No Brasil, a Lei 8.078 de 1990, o Código de Defesa do Consumidor não apresenta artigos que abordem as relações por meio da internet. As palavras "internet", "virtual" ou "aplicativo" sequer aparecem em todo o texto da lei. As decisões favoráveis aos usuários contra empresas de serviço virtuais são fundamentadas em jurisprudência baseadas na Doutrina do Direito.

Em estudo realizado anteriormente nos Estados Unidos, foram encontrados aplicativos com funcionalidades semelhantes aos encontrados neste estudo de monitoramento, registro e com informações para cuidadores. Todavia, encontraram aplicativos com a intenção de cuidar dos cuidadores, tendo como instrumentos utilizados para esse fim: grupo para suporte emocional, uso de escalas de mensuração de nível de estresse, sugestões para o cuidador apoiar a própria saúde com qualidade de vida e até conselhos para seus pares (REIS; NOVELLI; GUERRA, 2018).

Para os aplicativos com função de registro, recomendamos melhorias no controle de internações, notificação de consulta e exames, funcionalidade de carteira digital de vacinas com agendamento e lembretes da próxima vacinação. Neste contexto, Ali et al. (2019) defendem que, quando esses aplicativos estiverem sendo projetados, sejam implementados mecanismos personalizáveis para gerenciamento de lembretes que não sejam excessivos e inadequados. Além disso, para que não haja perda de dados, é importante sincronizar as informações em um sistema de nuvem.

Na escolha do aplicativo, é relevante observar a avaliação realizada por outros usuários. Ademais, seis aplicativos não apresentaram avaliação, comentários ou descrição de suas funcionalidades e nove apresentaram poucos arquivos 
baixados (downloads) ou não são atualizados há mais de um ano. Outros doze exigem cadastro para acesso e possuem reclamação dos usuários quanto às telas ou aos formulários em outras línguas com indisponibilidade de funções básicas e pouca usabilidade. Seria importante que aplicativos desenvolvidos em outras línguas fossem integralmente traduzidos ao português quando estes forem disponibilizados no Brasil.

Diante disso, destaca-se a necessidade do desenvolvimento de aplicativos que correspondam às necessidades dos cuidadores de idosos e seus familiares. Adicionalmente, sugere-se a necessidade de realização de novos estudos sobre a qualidade dos aplicativos móveis e sobre a fragilidade da legislação com relação ao uso deles. Este estudo teve como limitação a seleção de aplicativos mais abrangentes e a exclusão de aplicativos regionais. Sugerem-se novos estudos que avaliem os aplicativos de acordo com as regiões do Brasil e/ou por meio de entrevistas diretas aos usuários. O aprofundamento da discussão sobre a fragilidade da legislação com relação ao uso dos aplicativos móveis é também de vital importância. Dessa forma, que futuramente haja maior segurança legal aos usuários, profissionais e empresas desenvolvedoras de aplicativos móveis.

\section{Considerações finais}

Com base nos resultados deste estudo, foi possível concluir que os aplicativos móveis avaliados tiveram resultados positivos na avaliação (nota quatro ou mais) de seus recursos. Entretanto, é necessária a inclusão de novas funcionalidades e ajustes no que tange à melhoria da sua usabilidade. Verificou-se que há disponibilidade de aplicativos móveis com o propósito de auxiliar o cuidado ao idoso e promover aproximação entre familiares e o idoso assistido. Contudo, ainda não há implementação efetiva dos recursos tecnológicos (bate-papo, mensagem de texto, videochamadas, dentre outros) que possam concretamente proporcionar tal aproximação.

As principais indicações de melhorias nos aplicativos estão direcionadas ao controle de internações, notificação de consultas e exames, manejo das vacinas com agendamento e lembretes da próxima vacinação, back up dos dados e sincronização em nuvem (Internet) para garantir a segurança e o armazenamento dos dados. Recomenda-se ainda a inserção de mecanismos de verificação de antecedentes criminais, qualificação técnica do profissional, registro funcional, junto aos dados do profissional cadastrado na plataforma e carta de indicação do último contratante ou clientes. Tal funcionalidade possibilitará ao idoso e seu familiar maior segurança no momento da contratação do profissional cuidador de idoso. 
abstract

The aim of the study was to analyze mobile apps available on the Play Store and App Store platforms, focusing on caregivers of elders in Brazil. The apps will be in the Portuguese language, and also have instruments to assist the elderly and/or promote a commercial relationship between caregiver of the elderly and family. Only 28 out of 247 apps matched the research objectives and were organized into categories, dezoito apps are for bringing professionals or services together and family or older adults, seven are for assisting professional caregiver or family, three are for monitoring older adults; four are for recording the elderly's information and five belong to more than one category. There are apps available in stores with themes focused on the care of elders and promote the relationship between the family. However, there was a need to include new features and adjustments to improve apps.

keywords

Technology Assessment Biomedical. Mobile Applications. Caregivers.

referências

ALI, Eskinder Eshetu et al. Design Considerations in the Development of App-Based Oral Anticancer Medication Management Systems: a qualitative evaluation of pharmacists and patients perspectives. Journal of Medical Systems, v. 43, n. 3, p. 1-14, feb. 2019.

BARRA, Daniela Couto Carvalho et al. Métodos para Desenvolvimento de Aplicativos Móveis em Saúde: revisão integrativa da literatura. Texto \& Contexto - Enfermagem, v. 26, n. 4, p. 1-12, jan. 2018.

BRASIL. Lein. 8.078, de 11 de setembro de 1990. Disponível em: http://www.planalto. gov.br/ccivil_03/leis//8078.htm. Acesso em: 19 jun. 2020.

BRAVO, José et al. M-Health: lessons learned by m-experiences: Lessons Learned by m-Experiences. Sensors, v. 18, n. 5, p. 1569-1596, may 2018.

GARTNER. Gartner Says Worldwide Sales of Smartphones Recorded First Ever Decline During the Fourth Quarter of 2017. Gartner Consulting, 2018. Disponivel em: https:// www.gartner.com/en/newsroom/press-releases/2018-02-22-gartner-says-worldwidesales-of-smartphones-recorded-first-ever-decline-during-the-fourth-quarter-of-2017. Access in: 07 aug. 2019. 
GROSSMAN, Molli R; ZAK, Deanah Kim; ZELINSKI, Elizabeth M. Mobile Apps for Caregivers of Older Adults: quantitative content analysis. Jmir Mhealth And Uhealth, v. 6, n. 7, p. 162-178, july 2018

IBGE. Sinopse do Censo Demográfico 2010. Instituto Brasileiro de Geografia e Estatística - IBGE, Rio de Janeiro, 2010. Disponível em: http://biblioteca.ibge.gov.br/visualizacao/ livros/liv49230.pdf. Acesso: 22 jun. 2019

KIM, Hyunmin; POWELL, M. Paige; BHUYAN, Soumitra S. Seeking Medical Information Using Mobile Apps and the Internet: are family caregivers different from the general public? Journal Of Medical Systems, v. 41, n. 3, p. 1-8, jan. 2017.

MEIRELLES, Fernando S. Pesquisa Anual do Uso de TI. Fundação Getúlio Vargas. 30. ed. São Paulo, 2019. Disponível em: https://eaesp.fgv.br/sites/eaesp.fgv.br/files/ pesti2019fgvciappt_2019.pdf. Acesso em: 02 jul. 2019.

PHONGTANKUEL, Veerawat et al. Mobile Health Technology Is Here-But Are Hospice Informal Caregivers Receptive? American Journal of Hospice and Palliative Medicine, v. 35, n. 12, p. 1547-1552, jun. 2018.

QUINN, Charlene C. et al. Mobile Support for Older Adults and Their Caregivers: dyad usability study. Jmir Aging, v. 2, n. 1, p. e12276-12293, may 2019.

REIS, Edison dos; NOVELLI, Marcia Maria Pires Camargo; GUERRA, Ricardo Luís Fernandes. Intervenções realizadas com grupos de cuidadores de idosos com síndrome demencial: revisão sistemática. Cadernos Brasileiros de Terapia Ocupacional, v. 26, n. 3, p. 646-657, 2018.

SILVA, Bruno M.c et al. Mobile-health: a review of current state in 2015. Journal of Biomedical Informatics, v. 56, p. 265-272, aug. 2015.

WAISBOURD, Michael et al. The Wills Eye Glaucoma App. Journal Of Glaucoma, v. 25, n. 9, p. e787- e791, sept. 2016

WARPENIUS, Erika et al. A mobile user-interface for elderly care from the perspective of relatives. Informatics For Health And Social Care, v. 40, n. 2, p. 113-124, nov. 2014.

WILLIAMSON, Steven S; GORMAN, Paul. N; JIMISON, Holly. B. A Mobile/Web App for Long Distance Caregivers of Older Adults: Functional Requirements and Design Implications from a User Centered Design Process. Amia Annu Symposium Proceedings Archive, v. 2014, p. 1960-1969, nov. 2014

Data de Submissão: 19/05/2020

Data de Aprovação: 22/06/2020 